\title{
Emergence: Distracting Notions and How to Get It Right \\ Vincent Vesterby
}

\section{INTRODUCTION}

Most of the literature of emergence is replete with ideas that that do not relate to the intrinsic nature of emergence itself. It is not possible to achieve an accurate understanding of emergence and its roles in the universe when the mind is distracted by misleading notions.

The first half of this paper points out some of the distracting notions in the emergence literature, and explains why these extraneous ideas are not relevant to emergence. To begin, a short description of the nature of emergence is provided which serves as a context against which the inadequacy of these notions can be seen. There is next a list of some of the offending notions, which is then followed by examples and discussion.

The second half of the paper discusses the basic process of what is required to analyze an actual case of emergence.

\section{EMERGENCE}

The important thing about emergence is that it is creative.

Emergence is the coming into existence of patterns-of-material-organization as a consequence of motion. (Vesterby 2019) This is both the foundational form of emergence, and the core quality of every case of emergence, no matter how complex any particular case of emergence may be. As far as is known, all matter in the universe is in motion. Emergence, then, is ubiquitous - with the entire material universe continuously emerging.

There are two primary aspects of emergence - the event of an emergent product coming into existence and the prior process that leads to that event.

There is the actual event of coming into existence of the emergent pattern-of-materialorganization - the event of the existence of a newly created atom in a star, the event of the existence of a newly created protein molecule in a cell, the event of the existence of the first individual of a new species of organism in an ecosystem. Each event of the new existence of an emergent pattern-of-material-organization, whether of an atom, a protein, or a new species, is a consequence of a preceding process that culminates in that event of new existence - the creative process of emergence.

Emergence is a factor. It is something that exists and plays a role in the intrinsic nature of the situation in which it exists.

Emergence is an isomorphy. It is a factor that exists in and plays roles in the nature of two to many different situations. 
Isomorphies, such as emergence, can occur at all the levels of the hierarchic organization of material reality, from the level of elementary particles to the material universe as a whole. Galactic superclusters are isomorphies. It is emergence, based on combinatorialenhancement $^{1}$, that creates the hierarchic organization of the material universe. Isomorphies, such as emergence, can also occur from one to many times all across a level.

A factor, as an isomorphy, can develop-occurring in simpler forms in simpler situations where few other factors are playing roles (such as at lower levels), and occurring in more complex forms in more complex situations where more factors are playing roles (such as at higher levels). The factor emergence, as an isomorphy, develops. Emergence due to motion is the simplest form of emergence. Initially emergence develops due to the roles of universally-foundational factors such as transformation-point, combinatorialenhancement, adjacent-relation, push, and coherence. (For the foundational development of emergence see Vesterby 2019)

The intrinsic natures of higher level, more advanced stages in the development of emergence are due to the specific additional factors that are there playing roles in the process of emergence. The intrinsic qualities of something that exists determine the types of relations that something can have with other things that exist. Thus, various additional factors play their roles in the process of emergence according to what they are, according to their intrinsic qualities — which results in the different types of emergence.

Because the intrinsic qualities of something that exists determine the types of relations that something can have with other things that exist, the existence and intrinsic nature of what goes before determine the existence and intrinsic nature of what follows. The process of emergence determines the existence and intrinsic nature of the emergent product. Thus, the creative process of emergence is intrinsically determinate.

\section{DISTRACTING NOTIONS}

The general problem is that people often make statements about emergence that have more to do with how people relate to emergence than with the nature of emergence itself. For example, it is said that emergence is unpredictable, irreducible, or a theory.

${ }^{1}$ combinatorial-enhancement-Whenever two or more things exist at the same time, whenever they are coexistent, there will be some form of relation between them, minimally the spatial-relations of distance, direction, and positional-orientation.

Whenever coexistent things are combined in some manner, there will be new emergent relations. When they come near one another, patterns-of-material-organization emerge, such as group relation and hierarchic organization. When material objects move into adjacent-relation, wherein there is no space between them, supraorganizational factors emerge, such as contact, push, and coherent-relation.

These emergent factors, that are due to proximity or adjacent relation, are newly existing. They are new to the situation, additions to the situation-enhancements of the situation. They are enhancements due to combining - combinatorial-enhancements.

Combinatorial-enhancement is a form of development, a process of emergence - combinatorialenhancement-based-emergence. It is the basis of (a) the whole is greater than the sum of the parts, (b) emergent complexity, and (c) the hierarchic-organization of material-reality. 
Predicting, attempting to reduce, and theorizing are things humans do. They are not intrinsic qualities of emergence as it exists and plays its roles in creating the hierarchic organization of material reality throughout the universe, from elementary particles to galactic clusters.

Statements that have little or nothing to do with what emergence is probably come from a failure to examine a broad enough spectrum of actual cases of emergence across all the disciplines to achieve an accurate general understanding of the intrinsic nature of emergence.

\section{Distracting notions that occur in the literature of emergence}

1. The Initial Problem: George Henry Lewes-Focusing on the trivial rather than the substantial.

2. The notion that an emergent (quality, product, object, system, or level) must be novel.

3. The idea that emergence is a notion, a concept, or a theory.

4. The opinion that emergence is unpredictable.

5. The claim that an emergent product (quality, object, or system) does not occur at any lower level of the deep structure of the emergent product.

6. Inadequate definitions of emergence that result from limited viewpoints.

7. The notion that the idea of reduction is relevant to emergence.

8. The claim that resultant properties are not emergent properties.

\section{EXAMPLES AND DISCUSSION}

There are three websites that have articles providing broad coverage of the discussion about emergence. Most of the examples presented here are from these websites.

Wikipedia.

Internet Encyclopedia of Philosophy.

The Stanford Encyclopedia of Philosophy.

\section{The Initial Problem: George Henry Lewes-Focusing on the trivial rather than the substantial}

The problem started with George Henry Lewes in 1891 when he coined the term emergent. He applied this well-chosen term to the wrong part of the situation he was thinking about.

"Thus, although each effect is the resultant of its components, the product of its factors, we cannot always trace the steps of the process, so as to see in the product the mode of operation of each factor. In this latter case, I propose to call the effect an emergent." (Lewes 1891)

The statement, ". . . each effect is the resultant of its components, the product of its factors, ..." says something about the universe, something about its structure and processes. The statement, "... we cannot always trace the steps of the process, so as to see in the product the mode of operation of each factor." does not provide knowledge or 
understanding about the universe. It is just a statement of human ignorance - it does not merit a special label.

Lewes should have said - In the former case, I propose to call the effect an emergent.

Effects are the resultants of their components, the product of their factors, regardless of whether we can trace the steps of the process. All effects should be called emergent in order to draw attention to the importance of doing the necessary research to determine the steps of the process that result in the emergence of the effect.

Instead, Lewes coined a term for the ignorance relation between humans and the process that results in the emergence of the effect.

According to Lewes, if we do not know the process whereby an effect comes into existence, the effect is to be called emergent. If we do know the process, the effect is not called emergent. However, if knowledge of the origins of an emergent effect is then acquired, the effect is no longer emergent.

With the acquisition of the knowledge of the origin of the effect, nothing intrinsic to the effect or the processes of its origins has changed. The only change is in the state of human knowledge about the effect, which knowledge occurs in the mind, where it is entirely extrinsic to the effect itself and its origins. To call the effect itself emergent or not emergent based on the state of knowledge about the origins of the effect provides no information about the nature of the effect or its origins. With Lewes' proposal, the term refers to something extrinsic to the effect, something, knowledge or the lack thereof, which occurs in the human mind, rather than in the effect.

How and why things come into existence is a critically important issue to resolve when working towards an understanding of the intrinsic nature of reality - of that which exists - that is, the intrinsic nature of the universe and all that exists within it.

Fortunately, the terms emerge, emergent, and emergence can have the substantial meaning 'to come into existence'.

emerge 4. to come into existence; develop.

(Random House Unabridged Dictionary, 2nd. ed. 1993)

emergent 1. Emerging; coming into view or into existence; nascent; new.

(Wiktionary. https://en.wiktionary.org/wiki/emergent. 2018)

emergence the fact of something becoming known or starting to exist.

(Cambridge Dictionary.

https://dictionary.cambridge.org/dictionary/english/emergence)

Using these terms to refer to the coming into existence of an effect- the process and event of coming into existence-gives them a meaning that refers to something intrinsic to the effect. 
When Lewes labeled the relatively trivial part of the situation, the ignorance part, he created a distraction from the substantial part, that effects result from their components and associated factors. As it has turned out, this opened the floodgates for over a hundred years of distracting notions about emergence. During that time there has been little in the literature about emergence that seriously considered how and why the effect is the resultant of the components and their associated factors.

2. The notion that an emergent product (quality, object, system, level) must be novel The role of emergence in the universe is the creation, due to the motion of matter, of newly existing pattern-of-organization of material structure and process. Because essentially all matter in the universe is in motion, emergence is ubiquitous. The entire material universe is continuously emerging, with ongoing universal creation of new pattern-of-material-organization.

T. A. Goudge, in his article, "Morgan, C. Lloyd" (Goudge 1967), lists five criteria for emergents that were proposed by Morgan. The third one requires emergents to be novel. "An emergent. . . (3) is something genuinely new in the history of the universe, ..."

From the limited viewpoint of a human, from the limited position in space and time of a human, it is not possible to observe, demonstrate, or to figure out that something is genuinely new in the history of the universe. If this manner of being novel is a criterion for something to be emergent, then the term is vacuous.

The term novel can have less extravagant meanings.

"novel ... of a new kind; different from anything seen or known before" (Random House Unabridged Dictionary, 2nd. ed. 1993)

The usage that refers to the relation between a person and something they have never before seen or known does not refer to anything intrinsic to the thing or event that is called emergent. The term refers to the experience of the person only.

The simple claim that the emergent must be of a new kind does not necessarily carry the burden of universality. An emergent can be of a new kind in a few specific situations. A particular emergent may be novel in the situation in which it occurs, and also novel in a different situation at a different time or place. For example, the ability to roll up into an armored ball has evolved at least twice, once with a crustacean, the woodlouse (image at Wikipedia: woodlouse), and again with a myriapod, the giant pill millipede (image at Wikipedia: giant pill millipede).

However, having novelty in the form of new-kind as a criterion for emergence is an arbitrary criterion chosen by a person trying to characterize emergence. For example:

If we were pressed to give a definition of emergence, we could say that a property is emergent if it is a novel property of a system or an entity that arises when that system or entity has reached a certain level of complexity 
and that, even though it exists only insofar as the system or entity exists, it is distinct from the properties of the parts of the system from which it emerges. (Vintiadis)

A group of 27 cubes can be arranged in the form of a larger cube. Both the components and the whole have the property of being cubes. Both the smaller cubes and the larger cube have the quality of being cubes due to the spatial organization of their components. The smaller cubes are called emergent because they constitute the first time the cube form has occurred in the situation. The larger cube is not emergent according the criterion of novelty because it is a second occurrence of the cube form in the situation.

Saying the smaller cubes are emergent because they came into existence first in the situation, and saying the larger cube is not emergent because it came into existence second is an arbitrary choice, a notion that distracts from the observation that the cube form emerges twice in this situation.

\section{The idea that emergence is a notion, a concept, or a theory}

A concept about emergence is itself not the reality-referent of the concept.

Emergence is a process-pattern-of-organization that is a ubiquitous component of the universe. People can have ideas, notions, and theories about emergence, but these mental concepts are not emergence itself. When a person is thinking about emergence as a concept, an idea, notion, or theory, they are not thinking about emergence itself, they are not thinking about the reality-referent of the mental concept.

When a person is thinking about the qualities or components of a concept, the thinking does not need to go outside the mind because the concept exists within the mind. When thinking about the reality-referent of the concept, which exists outside the mind, the thinking must go there, outside the mind so to speak. While the mind uses concepts to think about emergence, the concepts must be about what is outside the mind, not about the concepts themselves. To think about what is outside the mind, about emergence itself, the concepts must have reality-referents. The concepts must be based on input from the senses.

An example of thinking of emergence as a theory is mentioned by Vintiadis, in the Internet Encyclopedia of Philosophy: 'In Lewes' work, three essential features of emergence are laid out. First, that emergentism is a theory about the structure of the natural world; ..." O'Connor and Wong, in The Stanford Encyclopedia of Philosophy, give an example from Mark Bedau (1997) of thinking of emergence as a notion: "He defines the notion of a weakly emergent state . . . thus: . . ." In their article, "Emergent Properties", in the Stanford encyclopedia, O'Connor and Wong provide an example of thinking about emergence as a concept: "Though details differ, representatives of this approach [epistemological emergence] characterize the concept of emergence strictly in terms of limits on human knowledge of complex systems." (O'Connor 2015) The Wikipedia article on emergence contains this sentence: "John Stuart Mill [3] and Julian 
Huxley [4] are two of many scientists and philosophers who have written on the concept."

The consequence of thinking about emergence as a mental entity - an idea, notion, concept, or theory - is that the discussion about emergence is not well guided by realitychecks, and tends to be overly complex and unrealistic.

\section{The opinion that emergence is unpredictable}

Prediction is something that humans do-it is not an intrinsic quality of emergence.

Because the process of emergence is determinate, the event of emergence can, in principle, be predicted - it all depends on (1) access to the relevant data, (2) understanding the data according to the dictates of structural-logic (see below), and (3) understanding that emergence occurs by way of stages and levels.

It often happens that a situation to be predicted is too small, too far away, too fast, or too complex, and the information for prediction is not available. The event of emergence in these situations cannot be predicted, but it is a matter of access to the relevant data, and not a matter of an intrinsic quality of the process of emergence. Under these circumstances, it is incorrect to claim that emergence is unpredictable by nature.

The intrinsic qualities of something that exists determine the types of relations that something can have with other things that exist such that the existence and intrinsic qualities of what goes before determine the existence and intrinsic qualities of what follows. With the process of emergence, the intrinsic qualities of the components of the situation, and the resulting types of interrelations, determine the existence and intrinsic nature of the emergent.

Structural-logic is the manner in which the intrinsic qualities of something that exists determine the relations that something can have with other things. With the process of emergence, it is structural-logic that determines the sequence of relations that result in the event of emergence. To be able to predict an emergent event, it is necessary to understand the structural-logic that occurs during the process of emergence. Any claim that emergence is unpredictable by nature ignores the role of structural-logic in determining the emergent event.

Goudge provides an example. He gives Morgan's fourth criteria for emergents. "An emergent ... (4) occurs in a manner that is unpredictable in principle since it conforms to no general laws, ..." (Goudge 1967, p.393)

The process of emergence progresses by stages and levels. It is not possible to predict developed stages and higher levels without including the determining roles of the emergent factors and structural-logic of the intervening stages and levels. To claim that emergence is unpredictable by nature because the determining roles of the intervening stages and levels are not being considered is unrealistic. 


\section{The claim that an emergent product (quality, object, or system) does not occur at any lower level of the deep structure of the emergent product}

Material-reality is hierarchically organized, level by level, with each higher level composed of all the levels below it all the way down to the foundational level of elementary particles. The series of levels from the level just below any particular level down to the foundational level of elementary particles constitutes the deep-structure of that particular level.

Emergence, based on combinatorial-enhancement, creates the hierarchic organization of the material component of the universe by combining elementary particles into the intrinsic patterns-of-organization of qualities, objects, systems, and levels. Thus, every quality, object, system, or level is composed of elementary particles - they are all emergent patterns-of-organization of groups of elementary particles.

To say that an emergent product does not also occur as a feature of any of its components is to say that the pattern-of-organization of the emergent product does not occur as a feature of the patterns-of-organization of the components of the emergent product. There is one way in which this is true, and multitudes of ways in which it is not true.

It is true in that the overall pattern-of-organization of the emergent product cannot occur as a feature of any of its components because it is composed of all of the components combined. None of the components, no lower level, is sufficient to form the entire pattern-of-organization of the top level emergent.

Nevertheless, it is not true that an emergent product cannot also occur as a feature of one or more of its components. While the top level emergent cannot occur in lower level components because it exists as all of them combined, and is thus too big to fit in a lower level, the pattern-of-organization of the top level can occur in lower level components as an isomorphy.

A simple example was given previously with the larger cube composed of 27 smaller cubes. The pattern-of-organization, the emergent quality, cube, of the larger cube also occurs with all 27 components. A complex example is emergence itself. Because the process of emergence creates all the levels of material-reality, it occurs at all the levels. A great many emergent patterns-of-organization of material structure and process can occur as isomorphies at two to many of the levels of the hierarchic organization of materialreality.

The occurrence of features of emergent products at multiple levels is so common throughout the universe that it can be used as the basis of a methodology for improving communication between the disciplines. For example, threshold situations occur in the subject matters of all the disciplines, that is, threshold occurs at all the different levels studied by the different disciplines. Understanding the nature and roles of threshold in one discipline enables improved understanding of other disciplines where threshold plays roles. Discipline-independent-transdisciplinarity finds these multilevel factors and integrates them into a transdisciplinary language. (Vesterby 2013) 
It is true that the top emergent level in any situation cannot have occurred at any lower level because it exists as all the lower levels combined. It is also true that emergence is by nature a creative process and often creates emergent products, emergent patterns-oforganization of material structure and process, that are novel to the situation, patterns that have not occurred in any of the lower levels in the deep-structure of an object, system, or level. Nevertheless, emergence also creates isomorphic emergent products, isomorphic patterns-of-organization such as cube, threshold, and emergence, that occur at two to many levels. It is this creation of isomorphies that renders false the idea that an emergent property never occurs at lower levels of the deep-structure of that emergent property.

In the Wikipedia article on Emergence there is an example of this distracting notion, which is a false defining criterion of emergence: "In philosophy, emergence is often understood to be a claim about the etiology of a system's properties. An emergent property of a system, in this context, is one that is not a property of any component of that system, but is still a feature of the system as a whole."

The Internet Encyclopedia of Philosophy article on emergence has an example: "Mario Bunge's 'rational emergentism' (1977) is a form of weak emergence according to which emergent properties are identified with systemic properties that none of the parts of the system share ...." (Vintiadis)

The New England Complex Systems Institute article on emergence begins with this error: "Emergence refers to the existence or formation of collective behaviors - what parts of a system do together that they would not do alone." An isomorphic pattern can occur both as a result of the collective behaviors of the parts of a system, and as a result of the collective behaviors of the parts of a subsystem of the overall system.

That emergent properties do not also occur at lower levels of the deep-structure of an emergent product is just an idea. Somebody thought it up — somebody imagined it. To project an imagined idea onto a situation where it does not fit is anthropomorphic.

\section{Inadequate definitions of emergence that result from limited viewpoints}

Emergence is ubiquitous in the universe. It occurs at every level of the hierarchic organization of the material-universe. It thereby occurs in the subject matters of all the disciplines. Emergence is an isomorphic factor-it develops. It occurs in simple forms in the simpler situations and levels, such as physics and chemistry, and in complex forms in complex situations and higher levels such as biology, sociology and ecology.

Trying to define emergence by way of only one or a few disciplines or levels can only result in inadequate misleading definitions.

The Stanford article on emergence points out the tendency to define from a limited viewpoint: "A general rule of thumb in perusing the diverse recent literature on emergence is that emergence encompasses whatever striking macroscopic phenomena the theorist in question is interested in. Philosophers desire the concept to have clear 
application, and their different foci within the special sciences lead them to slant the notion in somewhat distinct ways." Trying to define a general factor that occurs at all levels throughout the universe by way of limited interests or from the limited viewpoints of the special sciences is not scientific.

The Wikipedia article on emergence gives an example. "Economist Jeffrey Goldstein provided a current definition of emergence in the journal Emergence. Goldstein initially defined emergence as: "the arising of novel and coherent structures, patterns and properties during the process of self-organization in complex systems."'

Complexity is not a defining requirement of emergence. Emergence is the coming into existence of newly occurring pattern-of-organization of material structure and process. Foundationally, the emergence of a newly existing pattern-of-material-organization requires only two units of matter and the motion of one of them, with the motion resulting in the change of distance and/or direction relations between the two units and the emergence of a newly existing pattern.

\section{The notion that the idea of reduction is relevant to emergence}

The important thing about emergence is that it is creative.

Each emergent higher level is composed of all the lower levels of its deep-structure combined, from the foundational levels of elementary particles, atoms, and molecules, up through all the levels to the level where the combined components of that level form the object, system, or top level. Because each higher level is a combination of its lower levels, the higher level itself does not exist at any of the lower levels of its deep-structure. A higher level cannot be reduced to a lower level-its overall pattern-of-organization of components and relations is not, cannot be, at any lower level.

A simple, observable example is a crystal composed of molecules. All of the molecules of the crystal and all their interrelations combined constitute the top level, the crystal level. The individual molecules themselves constitute the molecular level, the level just below the top level, and are the uppermost level of the deep-structure. The next level down in the deep-structure of the crystal is the individual atoms that constitute each molecule. The next level down are the elementary particles that constitute each atom.

The pattern-of-organization of the crystal does not occur at the level of an individual molecule because the crystal pattern requires more than one molecule. The pattern-oforganization of a molecule does not occur at the level of an individual atom because the molecular pattern requires more than one atom. The pattern-of-organization of an atom does not occur at the level of an individual elementary particle, such as a proton, neutron, or an electron, because the pattern-of-organization of an atom requires more than one elementary particle.

However, it is possible for either lesser developed or more developed isomorphies of the top level pattern-of-organization to occur at lower or higher levels. But the higher level, developed, isomorphies, which occur as emergents, cannot be reduced to their lower 
level, less developed, forms because the more developed forms have additional factors playing roles in what they are.

While it is true that emergents cannot be reduced, it is pointless to say so. To include an extraneous notion like irreducibility as part of the defining characteristic of emergence is not only irrelevant, it detracts from the effort to find out what emergence really is.

The Emergence article on Wikipedia points out how common the reduction notion is in the emergence literature: "Almost all accounts of emergentism include a form of epistemic or ontological irreducibility to the lower levels."

The Wikipedia article on Emergentism has an example: "Emergent properties are not identical with, reducible to, or deducible from the other properties."

The article on emergence in the Internet Encyclopedia of Philosophy provides an example from Lewes: 'In Lewes' work, three essential features of emergence are laid out ... Third, that the question of emergence is related to the question of the possibility of reduction."

Then the article itself proceeds to indulge in this notion that the idea of reduction is relevant to emergence: "These three features will structure this article's discussion of emergence." Terms such as reduction, reducible, and irreducible are used 79 times throughout the article. Yet reduction in this sense has nothing to do with the intrinsic nature of the process or the event of emergence. The article is in large part not actually about emergence. It is not possible to learn much of anything about emergence in this article.

The Stanford Encyclopedia of Philosophy states this distracting notion in the first paragraph: "We might roughly characterize the shared meaning thus: emergent entities (properties or substances) 'arise' out of more fundamental entities and yet are 'novel' or 'irreducible' with respect to them." Terms relating to the issue of reduction are used 47 times throughout the article - the more time discussing reduction, the less time discussing emergence.

Reduction is a mental exercise the occurrence of which is extrinsic to the actual situation, the level which is supposed to be reduced. Reduction is not a process that occurs naturally within the hierarchic organization of material reality outside the mind, and is thus not relevant to emergence.

\section{The claim that resultant properties are not emergent properties}

Lewes pointed out the difference between emergent products that are simply consequences of adding or mixing components that are homogeneous and commensurable where there is little or no interaction among those components, and emergent products that are consequences of a combination of components where the interaction of the components plays a role in the intrinsic nature of the emergent products. The difference is real and constitutes a major stage in the development of 
emergence from simpler forms to more complex forms - the development of the process of emergence from adding or mixing noninteractive components to an interactive combination of components.

Both emergence due to adding or mixing and emergence due to interactive combination are stages in the form of emergence due to combinatorial-enhancement. With both, the components must initially come together to form the group of components that displays the emergent product.

Lewes was working from a limited viewpoint, being unaware of the foundational form of emergence - creation of newly existing pattern-of-material-organization due to motion. He did not know about emergence based on sequential-enhancement. While he knew that the roles of various factors, different co-operants, produced various emergents, he was unaware of emergence as an isomorphy that develops due to the roles of additional factors. Thus, he did not recognize that the difference between emergents due to addition and mixing and emergents due to interactive combining was a stage in the development of the process of emergence.

Down through the years others have followed Lewes' notion that resultant properties are not emergent properties. The Stanford Encyclopedia of Philosophy article Emergent Properties has a section on Samuel Alexander, which presents Alexander's view on this issue: "He is also adamant that, ..., the mental process is 'not merely neural' (p. 6), but 'something new, a fresh creation' (p. 7). It involves a 'distinctive quality' (p. 55) which emerges, rather than merely being resultant, from the neural process (p. 14)."

The Internet Encyclopedia of Philosophy article Emergence has a section on the notion of strong emergence, which makes this statement: "Also, numerical novelty, the fact that a property is instantiated for the first time, is not enough to make it strongly emergent for, again, that would make many resultant properties emergent, like the first time a specific shape or mass is instantiated in nature."

In Lewes' time it was relatively easy to trace the origin of added or mixed emergents to the togetherness of their components, and, at that time, it was relatively difficult or impossible to trace the origin of the emergent products of an interactive combination of components. Lewes wanted to base his distinction between the two modes of origin on the ability of humans to identify the details of the process of emergence, which is a factor extrinsic to and not relevant to the intrinsic natures of the two stages of the development of emergence.

\section{ANALYZING EMERGENCE: HOW TO GET IT RIGHT}

The analysis of a real-world instance of emergence has three required components.

- What to know beforehand.

- What to do.

- What to observe. 


\section{What to know beforehand}

- Structural-logic.

- The general intrinsic nature of emergence.

\section{Structural-logic}

Structural-logic is the intrinsic logic of reality.

Structural-logic is the manner in which the intrinsic qualities of something that exists determine the types of relations that something can have with other things that exist. Structural-logic is determinate. (Vesterby 2008)

The intrinsic qualities of the various components of a situation determine the types of relations those components can have with one another. The intrinsic qualities of the emergent relations within that situation then determine the emergent overall pattern-oforganization of the situation. The manner in which the intrinsic qualities of the emergent relations determine the pattern-of-organization of the situation is determinate.

In the process of emergence, structural-logic determines both the types of relations and the sequences of relations. In the process of emergence, the existence and intrinsic qualities of what goes before determine the existence and intrinsic qualities of what follows. Higher levels developmentally emerge from the lower levels by way of the structural-logic of combinatorial-enhancement.

\section{The general intrinsic nature of emergence}

Emergence is the process whereby structural and process patterns-of-materialorganization come into existence as a consequence of motion

This is both the foundational form of emergence, and the core nature of every case of emergence, no matter how complex any particular case of emergence may be due to the roles of additional factors other than motion. The basic set of relations - emergent pattern due to motion - is always there giving each case of emergence its intrinsic-identity as an instance of emergence. The analysis of complex cases of emergence will reveal many distinct roles of motion distributed among the various factors playing roles in the process of emergence. Emergence cannot happen without the occurrence, without the role, of motion. However, if there is motion, there will be emergence. Because motion is universal, emergence is universal.

\section{The process and the event of emergence are intrinsically determinate}

The intrinsic qualities of the material components that play roles in the process of emergence determine the types of relations those components can have with one another. The types of relations and the intrinsic qualities of those relations then determine the emergent pattern-of-organization. Overall, the existence and intrinsic nature of the components and relations that have gone before in the process of emergence determine the existence and intrinsic nature of the following part of the process. The process of emergence determines the existence and intrinsic nature of the emergent product. 
The process of emergence is creative

Patterns-of-material-organization come into existence - they are created by the process. With emergence based on sequential-enhancement, patterns-of-organization are sequentially created by sequentially occurring motion rearranging the components of a situation relative to one another. With emergence based on combinatorial-enhancement, patterns-of-organization are created by motion combining components, which results in the emergence of groups and hierarchic organization. With emergence based on separation-enhancement, newly existing patterns-of-organization are created by motion that disperses units of matter, as with a salt dissolving in water, or with dung beetles rolling balls of dung away from a source and depositing them in their underground brood chambers, or with the extremely complex dispersal of matter resulting from a nova. In each of these examples, there are additional factors playing roles in the nature of the process of emergence, such as the factors that initiate the dispersal and the factors that determine the manner of dispersal - the Brownian motion of the molecules in the solution, the instinct driven behavior of the beetles, and the force of the explosion. Whether it is just rearranging components, or combining them, or dispersing them, there is always creation of newly existent emergent pattern-of-material-organization.

\section{Emergence is a factor}

A factor is something that exists in a situation and plays a role in the intrinsic nature of that situation. Because motion is universal, making emergence ubiquitous, emergence occurs in and plays roles in the nature of all situations. The understanding of all situations can be enhanced by understanding the role of emergence as a factor in those situations.

\section{Emergence is an isomorphy}

An isomorphy is a factor that exists in and plays roles in the intrinsic nature of two to many different situations. Because emergence occurs in and plays roles in the nature of all material situations throughout the universe, emergence is a universal isomorphy. Because it is emergence based on combinatorial-enhancement that creates the hierarchic organization of material-reality, isomorphic forms of emergence occur at all the levels. Isomorphies can be used as intellectual tools to establish a foothold of understanding in unknown situations. What is known about an isomorphy in one situation can be used to enhance the understanding of other situations where that isomorphy occurs. Because emergence is a universal isomorphy, understanding the general intrinsic nature of emergence will enhance the understanding of all situations - understanding emergence provides the mind with a conceptual foothold in strange or unknown situations.

\section{Emergence develops}

It occurs in simpler forms in simpler situations where few other factors are playing roles, and in more complex forms in more complex situations where more factors are playing roles. In all developed forms, the core quality - the creation of newly existing pattern of material organization due to motion - is always there, giving the developed forms their intrinsic-identity as forms of emergence.

The paper, The Intrinsic Nature of Emergence-With Illustrations (Vesterby 2019), discusses the foundational stages of the development of emergence. It becomes evident in 
this foundational development that emergent factors can play roles in the further development of emergence. The higher the level in the hierarchic organization of material reality, the more likely the factors that play roles in developed forms of emergence will themselves be factors that originated by way of emergence.

Emergence develops due to the additional factors playing roles in the process. The roles of additional factors can change the form of the process of emergence. Nonetheless, the core quality of emergence is always present in these developed forms, making them isomorphies of the factor emergence. Increasing complexity of isomorphic forms of a factor such as emergence is a form of development-factor development.

Development is transition with sequential-difference and enhancement. "Everything that exists takes part in one way or another in a universally omnipresent transition, a sequential-difference from one time, place, part, pattern, level, condition, stage, or situation to another involving some form of enhancement." (Vesterby 2013) Development occurs in many different forms. The process of emergence is one of these forms, with the enhancement being the creation of a newly existing pattern-of-materialorganization.

The process of emergence itself occurs as continuous ongoing emergence It is a process - a situation with ongoing change - with what follows a consequence of what has gone before. What follows emerges from what has gone before.

The ongoing process takes time to occur - the ongoing change occurs sequentially with ongoing time. The ongoing process exists sequentially with ongoing time. With an existing process of emergence, the ongoing sequence of change during time is the existential-pathway of that process of emergence-the specific existing sequence changes as the process progresses.

A situation is a combination of interrelated factors. A factor is something that exists, and plays a role in the origin, existence, structure, or processes of the situation or system in which it exists. The sequence of what a factor does, such as self-organization, and what happens to the factor, such as interactions with other factors, takes time to occur. The ongoing sequence of what a factor does and what happens to it during time is the existential-pathway of that factor - the specific existing sequence of events in the continuing-existence of that factor during time.

Because a situation is a combination of interrelated factors, the existential-pathway of a situation is the combination of the interrelated existential-pathways of its constituent factors. A process of emergence occurs as the combination of the interrelated existentialpathways of the components that play roles in that process of emergence. An emergent product is the consequence of the interrelated existential-pathways. It is this combination of the interrelated existential-pathways of the components that is to be mapped in the next section, What to Do. 


\section{What to Do}

- The prime imperative of analysis.

- Use science.

- Use structural-logic.

- Map the process of emergence and diagram the emergent product.

\section{The prime imperative of analysis}

To achieve an understanding of what emergence is, it is necessary to follow the prime imperative of analysis - Look to the subject of investigation itself. Let the intrinsic nature of the subject of investigation dictate to the mind the understanding of what it is.

Observe emergence, and describe what is observed. Describe carefully and fully, without any form of interpretation. Avoid including suppositions, preconceptions, and speculations - no hypothesis or theory. The use of the prime imperative involves only objective observation and objective description.

\section{Use science}

Science is a procedure, based on observation, that is designed to provide accurate knowledge and understanding about the intrinsic nature of reality, the intrinsic nature of that which exists. The scientific method is designed to enable careful objective observation resulting in objective knowledge and understanding. The method consists of a specific stepwise procedure.

- Observe first.

Science is based on observation - it is based on application of the prime imperative of analysis. The first step in the analysis of something that exists is to look at it.

- Second, speculate about what knowledge is still missing after observation (make hypothesis).

Base the hypothesis about what is not yet known about what was first observed. The hypothesis must be relevant to the subject of investigation. It must be relevant to what knowledge is missing after observation.

- Check speculation (make further observations, such as experiments, to check the validity of the hypothesis).

- Double check.

- Results should be repeatable, demonstrable.

- Repeat the previous steps until a body of facts has been accumulated (scientific knowledge).

- Summarize the body of facts into a coherent, integrated body of knowledge (scientific knowledge).

- Speculate about what knowledge and understandings are still missing from the summarized body of knowledge (summarized body of knowledge plus the associated speculation constitutes scientific theory).

- Check and double check theory-based speculation. 
- Repeat to continue accumulating the body of scientific knowledge and to develop the theory as a tool to indicate what to investigate next.

\section{Use structural-logic}

Structural-logic determines the manner in which the intrinsic qualities of something that exists determine the types of relations that something can have with other things that exist. Structural-logic determines the manner in which the existence and intrinsic qualities of what goes before determines the existence and intrinsic qualities of what follows. (For more on structural-logic see Vesterby 2008) In the process of emergence, structural-logic determines:

- the manner in which the particular components that play roles in a specific case of emergence determine the types of relations those components have with one another,

- the manner in which the relations that emerge between the components determine the pattern-of-organization of the group of components,

- the manner in which the relations that have gone before in the process of emergence determine what relations follow and the specific sequence of those following relations as the process of emergence progresses toward the emergent product, and

- the manner in which higher levels emerge from lower levels.

It is structural-logic that determines the existence and intrinsic nature of the emergent product.

To use structural-logic for the analysis of emergence, for the analysis of both the process and the event, it is necessary to first identify the components that play roles in emergence. And then to examine them to identify what intrinsic qualities they have and precisely how those qualities determine the types of roles the components can play with other components. A good way to draw the mind's attention to the intrinsic qualities of a particular component is to follow the method used in biology when studying a new species of organism - describe it in detail and draw it. The resulting images can then be used as components of the next stage in what to do-mapping the process.

\section{Map the process of emergence and diagram the emergent product}

The process of emergence consists of a series of relations, changes, events occurring during time. While some of these relations are concurrent, existing or occurring together at the same part of the process, others are not concurrent, instead occurring at separate parts of the process. To get an adequate overview of a particular process of emergence, which results in a particular emergent product, it is necessary to map the process, to diagram the sequence of stages of the process as they occur in time. The stages of the process must be spread out in a manner that displays their temporal sequence - spreading out the development of the process of emergence from its initiation to the event of the emergent product.

Notes that state the roles of the structural-logic that determines the relations and changes that occur at specific locations in the map should be placed in the map at those locations. 
A map that spreads out the development of a particular process of emergence depicts both the components playing roles in the process and the continuing-existence of these components during the ongoing progression of the process. The components occur in the map as images such as pictures or diagrams. The continuing-existence of the components is represented by generally horizontal lines from left to right through the map. In the map, the continuing-existence lines are differentiated by color, by line formatting such as dots or dashes, and by line thickness.

When various components are not interacting, the lines of their continuing-existence in the process run parallel to one another. When components interact, their continuingexistence lines converge to meet one another. When the components combine coherently, the continuing-existence lines run parallel and adjacent. When the components interact only briefly, the continuing-existence lines meet and then veer away from one another. (Figure 1)

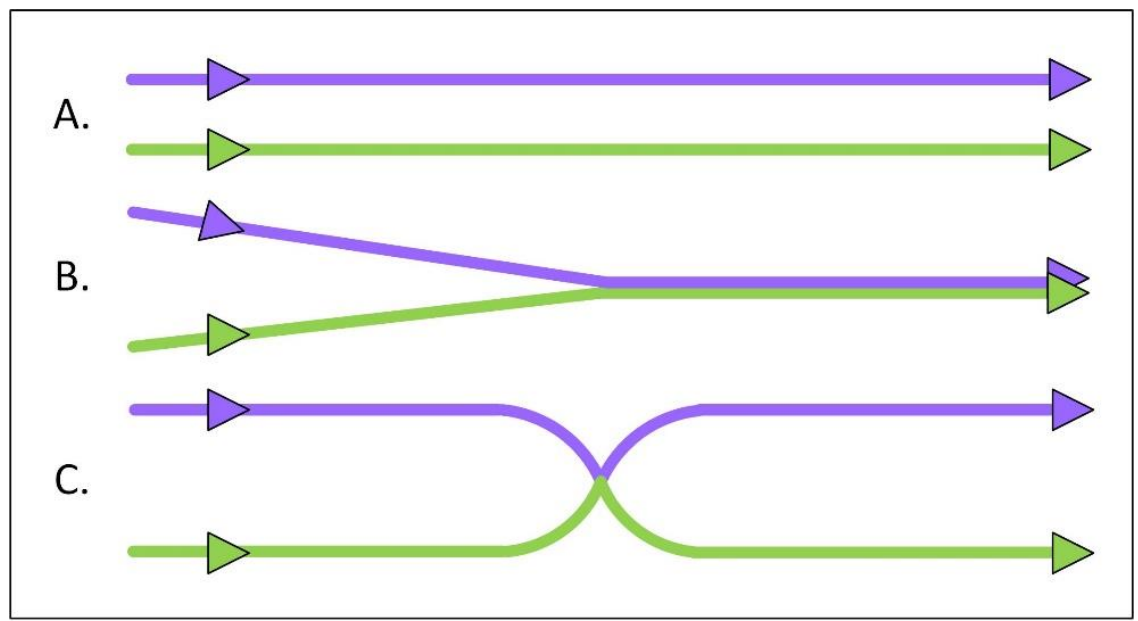

Figure 1. Some basic existential-pathway interactions: A. components not interacting, B. sequential enhancement of converging pathways progressing to combinatorial-enhancement and ongoing situation pathway, C. components interact only briefly.

There are a great many different ways in which the components of a process of emergence and their existential-paths can interact and influence one another. In some cases, components come in and join in coherent relation with another component, which results in various changes to the components (Figure 2).

In other cases, an incoming component interacts only momentarily with another component, or only for a short while, before separating and leaving. In these cases, the incoming component can be unchanged when it leaves, while the other component can be altered in various ways by the encounter (Figure 3 ). 


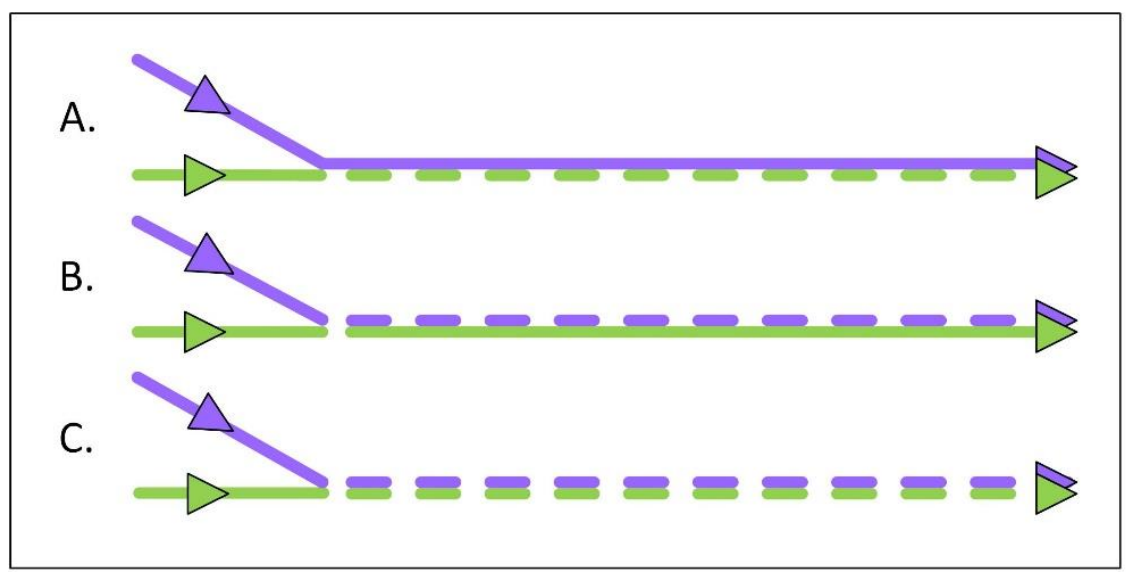

Figure 2. Two pathways combine resulting in A. a change of one pathway, or $\mathrm{B}$. a change of the other pathway, or $\mathrm{C}$. a change in both pathways.

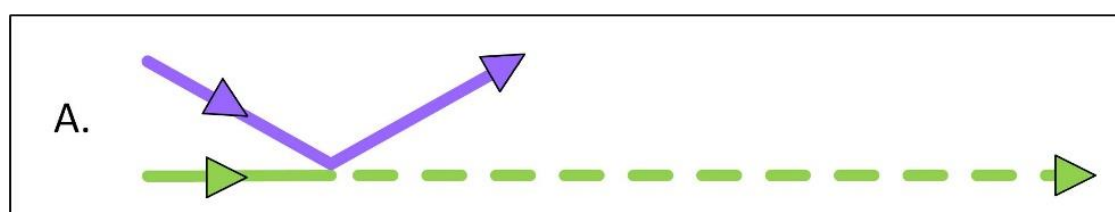

Two pathways interact momentarily. One separates and the other pathway changes.

B.

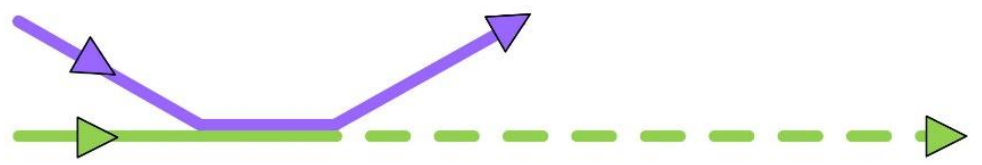

Two pathways combine in a coherent interactive relation for a while. One separates and the other pathway changes.

C.

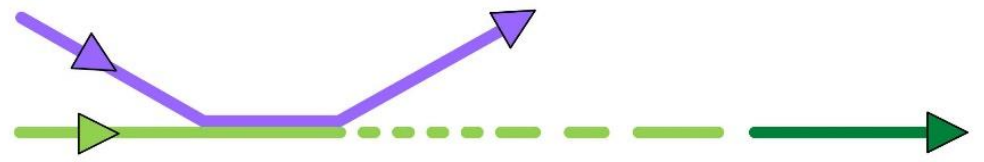

Two pathways combine in a coherent interactive relation for a while. One separates and the other has self-organization progressing to a major change.

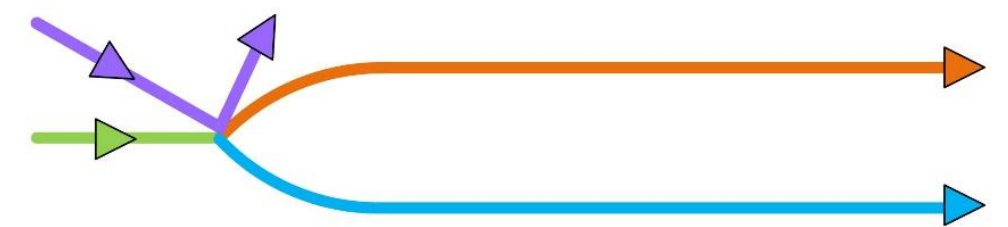

Two pathways interact momentatily. One separates and the other pathway divides.

Figure 3. Examples of pathway interactions where an extrinsic pathway comes in and causally interacts with another pathway, initiates change in that pathway, and then leaves. 
When an incoming component interacts with a coherent situation pathway, the consequence can be the decomposition of the coherent situation into its components, and various changes to the resulting individual pathways (Figure 4).

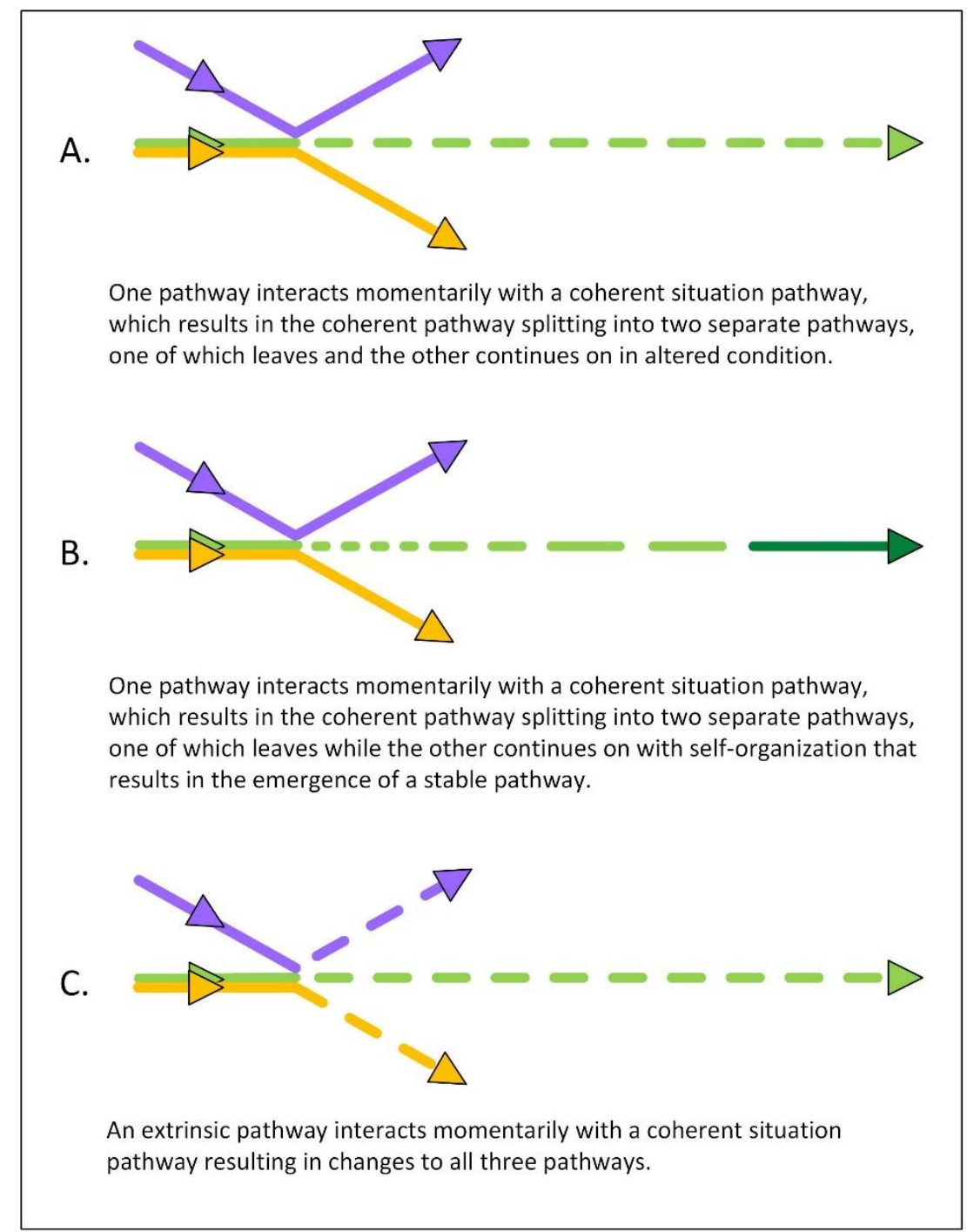

Figure 4. Examples of an incoming pathway interacting with a coherent situation pathway, resulting in the decomposition of the coherent pathway.

Changes to the existential-pathways playing roles in a process of emergence can be initiated by progressive self-organization within the pathways. Self-organization itself can be initiated by components combining into coherent situation pathways (Figure 5).

These examples of component existential-pathways that play roles in the process of emergence represent only a few of the vast number of possible types of component pathways and their interactions. 


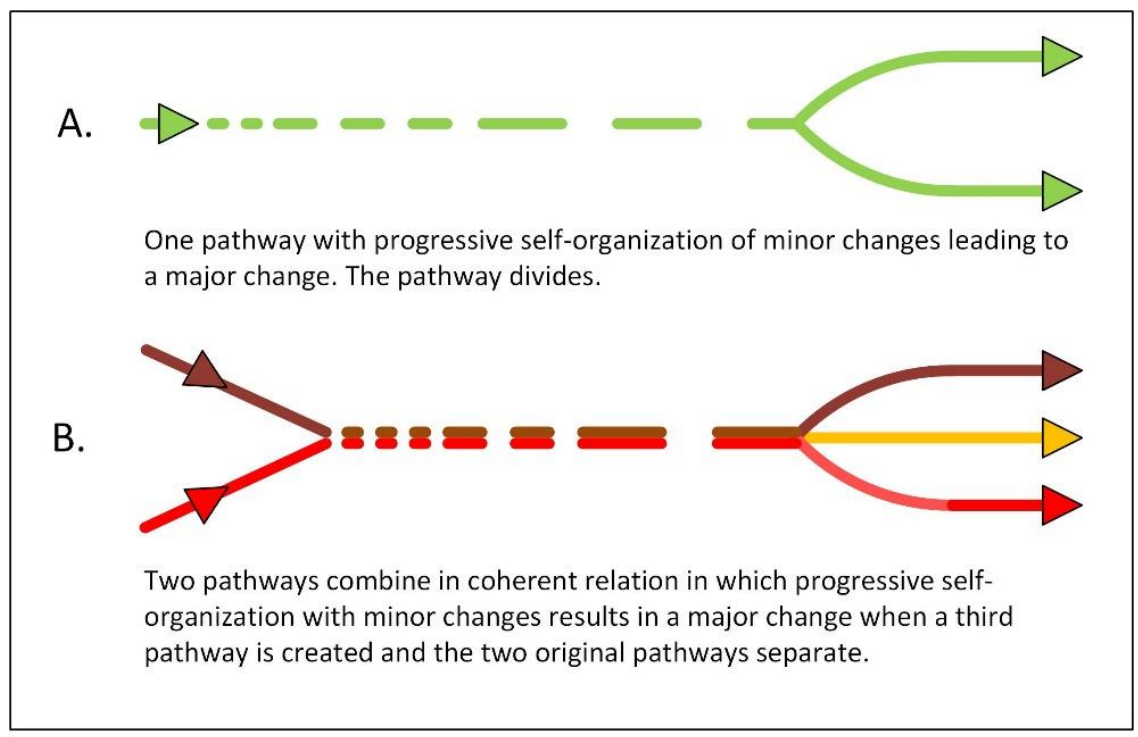

Fig. 5. More pathways with progressive self-organization.

At those points in the process of emergence, as depicted in the map, where the components interact, the components generally undergo some type of change. An image of the changed component(s) should occur at the location of the interaction. This is also where there should be a note about the structural-logic that plays a role in the change. There can also be notes throughout discussing what is going on in the process. A map of the process of emergence will have a few to many interacting continuing-existence lines, depending on how many components are involved in the process.

Such a map displaying the spread out progressive development, over time, of a process of emergence provides a comprehensive understanding of how and why the emergent product comes into existence. Then, at the end of the map, there should be a diagram of the emergent product showing the components and their interrelations.

\section{What to Observe}

- Components.

- Relations.

\section{Components}

Emergence is a process - a situation with ongoing change-with what follows a consequence of what has gone before. A situation is composed of components and their interrelations. The natures of the components, the intrinsic qualities of the components, determine the existence and natures of the relations. The intrinsic qualities of the relations determine the nature of the overall pattern-of-organization of the emergent whole. Within the process of emergence there are structural relations, existing in and organized in relation to space, and there are process relations occurring with and organized in relation to time.

Because there is the sequence, (1) the qualities of the components determine the existence and types of relations, and (2) the qualities of the relations determine the existence and 
nature of the emergent whole, the components constitute the foundation or core aspect of the process of emergence. The analysis of emergence starts there, with the components. The first requirement is to identify what components actually play roles in the process of emergence.

Every material component is foundationally composed of elementary particles. These elementary particles are combined into atoms, which in turn are combined into molecules. And the molecules combine into a vast diversity of materials, objects, and systems.

While every component from the elementary particles and molecules, to the objects and subsystems, are all playing roles in the nature of the situation, in the nature of the process of emergence, the great majority of those components are playing their roles far down in the deep-structure of the process situation.

For the analysis of a particular case of emergence, the components of interest are those that play direct roles in that process of emergence. These components that play direct roles can come from any level of the hierarchic organization of material-reality. Photons play roles in photosynthesis and vision. Molecules play roles in the synthesis of proteins, and proteins play roles throughout the structures and processes of the body. Muscles as organs, as subsystems, play roles in the motion of animals.

Once the specific components, whose roles determine what happens in a process of emergence, are identified, there are several things to note about each component. What are the intrinsic qualities of each component? Where does the component occur within the process? What is its existential-pathway through the process?

Was the component a component of the initial situation? If it was a component of the initial situation, what happens to its existential-pathway through the process of emergence? Does its existential-pathway extend all the way through the process of emergence to the emergent product? Does the existential-pathway terminate within the process because the component has been separated into its subcomponents? Does the existential-pathway interact with that of another component, and then veer out of the process situation?

Was the component initially outside the process, in the environment of the process, and entered the process somewhere along the existential-pathway of the process? Did this component enter the process, interact with a component in the process, and then leave the process, returning to the environment? Did this component enter the process, interact with a component in the process, and then remain within the process? Or did it enter the process and terminate there due to the separation of its subcomponents?

Did the component originate somewhere within the process due to the interactions of other components? What happens along the existential-pathway of that component? Did it interact with other components? Did it become a component of the final emergent product? 
Existential-pathways of components through the process of emergence can thus have various forms or configurations. They can continue independently through the process. They can interact with other components, either momentarily or continuously when the pathways combine. And combined pathways can separate, with components or subcomponents going their separate ways through the process.

When components interact, they usually influence or alter one another. The existentialpathways of components can thus have stages, such as the stage before an interaction and the stage after the interaction. Interactions initiate stages of existential-pathways.

\section{Self-organization in the process of emergence}

The process of emergence is a situation with ongoing change. The changing situation is composed of the existential-pathways of its components - situation-existential-pathway. The process of emergence is a type of situation-existential-pathway.

Sometimes a component-existential-pathway will continue without any internal change to the component. The component undergoes change only when, along its existentialpathway, it interacts with some other component. Other components can have enough internal complexity to be undergoing some form of internal change during their existential-pathways without any interaction with extrinsic factors, without interaction with other components. This is self-organization.

In general, self-organization occurs when factors intrinsic to a prior stage of development determine organizational factors intrinsic to the following stage, and do so without interaction with or influence from extrinsic factors. In component-existential-pathway, factors intrinsic to a prior part of that existential-pathway determine organizational factors intrinsic to the following part of that existential-pathway without influence of any kind from factors extrinsic to that existential-pathway. With self-organization in the situation-existential-pathway of the process of emergence, factors intrinsic to a prior part of that situation-existential-pathway determine organizational factors intrinsic to the following part of that situation-existential-pathway without influence from extrinsic factors.

The process of emergence can consist entirely of self-organization. Or it can be mostly or partly self-organization depending on the amount of extrinsic input.

It is structural-logic that determines what happens with self-organization in the process of emergence. Figuring out the structural-logic enhances the understanding of the selforganization that occurs in the process.

\section{Relations}

An emergent product exists as a pattern of relations between its components. The nature of the relations is a consequence of the nature of the components. After the intrinsic qualities of the components have been identified, the next step is to identify which of 
those qualities play roles determining the relations that are occurring between the components.

All components from atoms to galactic clusters are constituted of hierarchic organizations of subcomponents. The hierarchic organization of subcomponents constitutes the deepstructure of a component. The significance of this for the analysis of the process of emergence is that the interactions and connections between the components of the process can occur at any level of the hierarchic organization of the deep structure of the components.

An example of deep-structure connection between components occurs when two separate molecules combine into a single unit. The interaction between the molecules occurs between their subcomponents, at the atomic level, between the atoms of the two molecules. Another example occurs with a moving herd of bison. Each individual animal uses a subsystem, its visual system (an organ system) to see the other bison. The visual system subsystem of one bison detects the other bison as whole animals in order to coordinate with their movement. A third example of components interrelating with each other by way of deep-structure subcomponents occurs between persons. A person's mind is a subsystem of that person and can interrelate, communicate, with the equivalent subsystem, the mind, of another person. But these two subsystems do not interrelate directly. A language subsystem, a speech subsystem, and a hearing subsystem are used to communicate what is going on in one mind over to the other mind. Two subsystems, two minds, use other subsystems to establish a relation between the two minds, and thereby a relation between the two persons.

The actual nature of a relation between components provides the understanding of both how and why that relation emerges. An emergent product exists as a pattern of relations between its components. Understanding the actual nature of the relations that constitute the pattern-of-organization of an emergent product provides understanding of how and why that particular pattern-of-organization comes into existence-it provides understanding of how and why the emergent product comes into existence.

\section{ADDITIONAL PAPERS ABOUT EMERGENCE}

Vesterby, Vincent. 2019. "Emergence Is an Isomorphy." OSF Preprints. July 4. doi:10.31219/osf.io/ys3wd. https://osf.io/ys $3 w d /$

Vesterby, Vincent. 2019. "Emergence Is Why It Is Not Possible to Explain Life Solely with Physics and Chemistry.” OSF Preprints. July 9. osf.io/cwnt7. https://osf.io/cwnt7/

\section{REFERENCES}

Bedau, Mark 1997. "Weak Emergence," Philosophical Perspectives, 11: Mind, Causation, and World, Oxford: Blackwell, pp. 375-399.

Bunge, Mario. 1977. "Emergence and the Mind". Neuroscience 2: 501-509.

Goudge, T. A. 1967. "Morgan, C. Lloyd." In The Encyclopedia of Philosophy, v. 5. Macmillan Pub. Co. \& The Free Press; New York. 
Lewes, G. H. (1891) Problems of Life and Mind. First Series, The Foundations of a Creed. Vol. 2., Houghton, Mifflin and Co., Boston.

O'Connor, Timothy and Wong, Hong Yu. 2015. "Emergent Properties," in The Stanford Encyclopedia of Philosophy. https://plato.stanford.edu/archives/sum2015/entries/properties-emergent/

Vesterby, Vincent. 2008. The Origin and Foundational Development of Structural Logic. In Cook, D (ed.). Proceedings of the 14th ANZSYS Conference. Perth: School of Computer and Information Science, Edith Cowan University [CDROM]. ISBN 978-0-7298-0668-8 https://www.researchgate.net/publication/265143528_The_Origin_and_Foundatio nal_Development_of_Structural_Logic

Vesterby, Vincent. 2013. Discipline-Independent-Transdisciplinarity: The Essentials. https://www.researchgate.net/publication/299397879_Discipline-IndependentTransdisciplinarity The Essentials

Vesterby, Vincent. 2019. "The Intrinsic Nature of Emergence-with Illustrations." OSF Preprints. July 9. osf.io/n2skj. https://osf.io/n2skj/

Vintiadis, Elly. "Emergence." Internet Encyclopedia of Philosophy. http://www.iep.utm.edu/

“Emergence." Wikipedia.

Date of original version of "Emergence: Distracting Notions and How to Get It Right" May 2018. 